\title{
ARTICLE
}

\section{Agreement and bias in the estimation of fat free mass using bioelectrical impedance analysis and a resistance and reac- tance-based formula for the Mexican adults: A study from Merida, Yucatan}

\author{
Sudip Datta Banik \\ Department of Human Ecology, Cinvestav del IPN, Merida, Yucatan, Mexico
}

\begin{abstract}
Bioelectrical impedance analysis (BIA) is used to estimate body composition characteristics. The values of body fat and fat free mass (FFM) are obtained as per algorithms of the device that are often unknown to the researchers. Some models of the analyzer provide resistance and reactance values that may be useful to estimate FFM. Objective of the present study was to test the agreement and proportional bias in the estimation of FFM obtained through BIA and that derived from the resistance and reactance values using a formula for Mexican adults. A cross-sectional study was carried out in 2019 selected 60 university male students aged 21 to 23 years from Merida, Yucatan. A multifrequency whole body bioelectrical impedance analyzer Tanita MC 180 MA (Tanita Corporation, Tokyo-Japan) was used to evaluate body composition characteristics. The device gives estimates of FFM in $\mathrm{kg}$ (based on algorithm) and the resistance and reactance values (ohms). There is an existing formula for Mexican adults to estimate FFM (FFM_FOR) from the resistance and reactance values obtained through BIA. An agreement between the two estimates of FFM has been tested using Bland-Altman plot and linear regression analysis. Mean value of age of the participants was 21.88 years. FFM estimated by BIA (FFM_BIA = 41.44 kg) and that derived from FFM_FOR (41.36 kg) had significant intraclass correlation coefficient (ICC) (Cronbach's alpha $=0.99, p<0.0001)$. One sample t-test estimating the difference of mean values between FFM_BIA and FFM_FOR was not significant $(t=1.37$, mean difference $-0.02, p=0.18$ ). The Bland-Altman plot shows almost all data points lie within $95 \%$ confidence interval limits. A linear regression analysis using the difference of FFM values as dependent variable and the average of the measurements as the independent variable showed no significant interrelationships. In conclusion, the formula to estimate FFM using the resistance and reactance values of BIA has been found to be useful in the present study.
\end{abstract}

Acta Biol Szeged 64(2):91-98 (2020)

\section{KEY WORDS}

bioelectrical impedance analysis Bland-Altman intraclass correlation reactance regression resistance

\section{ARTICLE INFORMATION}

Submitted

11 October 2020.

Accepted

02 November 2020

*Corresponding author

E-mail: dattabanik@cinvestav.mx

\section{Introduction}

Evaluation of body composition characteristics is important in public health nutrition research. The last National Health and Nutrition Survey, 2012 (ENSANUT in Spanish acronym) reported high prevalence of obesity and related disorders in Mexican populations, particularly among adults (Gutiérrez et al. 2013). Remarkably high prevalence of overweight (39.6\%) and obesity (31.6\%) among adults from the southern region of the country have been reported in the survey. Yucatan is one of the Southern Peninsular States of Mexico where prevalence of excess weight (overweight and obesity) was very high (80\%) among adults that exceeded the national average (71.3\%). Prevalence of obesity was higher in the urban regions (34\%) than in the rural areas (26.5\%) of Yucatan (Gutiérrez et al. 2013).

Nutritional status assessment procedures are summarized by the mnemonic ABCD: anthropometric measurements, biochemical parameters, clinical diagnosis, and dietary habits (Dwyer et al. 1993). In anthropometric evaluation of nutritional status and body fatness using skinfold caliper, measurement errors could be high among excess weight individuals, particularly among centrally obese people (Sebo et al. 2017). Despite some limitations on the assumption of hydration factor and body geometry of severely obese individuals, bioelectrical impedance analysis (BIA) is reliable to evaluate body composition characteristics (body fat and fat free mass) with less intraobserver error than anthropometry (Coppini et al. 2005; Macias et al. 2007; Yamashita et al. 2012). The BIA is a 
simple and non-invasive technique; the portable equipment is relatively non expensive and provides accurate results (Bohm and Heitmann 2013; Ricciardi and Talbot 2007). The bioelectrical impedance (BI) instruments are different in number of electrodes and the models have in-built software programs (specific algorithm of the device that are unknown to the researcher) to estimate body composition characteristics by age, sex, and activity patterns etc. Several models of BI instruments are available in the market and quality assessment and validation are very important (Macias et al. 2007; Ramírez et al. 2012). The results obtained using BIA are also required to be validated with reference to the results obtained through dual-energy X-ray absorptiometry (DEXA or DXA) that is often considered as a gold standard for the evaluation of body composition characteristics (Khalil et al. 2014; Scafoglieri and Clarys 2018).

In this background, instead of using the direct values of body composition characteristics obtained from the BIA, Macias et al. (2007) developed a prediction equation to estimate fat free mass (FFM) for the Mexican adults using the resistance and reactance (ohms) values obtained through BIA and cross-validated the proposed equation with reference to the results obtained through air displacement plethysmography (ADP). In that study, the participants were 20 to 50 -year-old men and women from Sonora, a northern State in Mexico. The authors reported that the equation, based on two-compartment model was accurate, precise, and free of bias. They also reviewed the literature to justify that ADP was a reliable and valid method and as good as hydrostatic weighing for the evaluation of body composition characteristics. Till date, the prediction formula developed by Macias et al. (2007) is a reliable one that is available in Mexico to estimate FFM of adult individuals from the resistance and reactance values obtained through BIA and the article has been cited by many authors stating the usefulness of the formula (Balas-Nakash et al. 2010; Caicedo-Eraso et al. 2019; Lu et al. 2016; Mbada et al. 2015; Schifferli et al. 2011; Schifferli Castro and Zuniga 2017; Wada and Tekin 2007). This raised my interest to use the same formula for Mexican adults (Macias et al. 2007) and to find the agreement and bias between the estimated values of FFM (BIA-based and resistance and reactance-based) in a sample of adults from Merida, Yucatan, Mexico.

The objectives of the present study were to estimate body composition characteristics (body fat mass and fat free mass) of young adult men using the BIA, and to test the agreement and proportional bias in the estimation of fat free mass (FFM) obtained through BIA and that derived from the resistance and reactance values using in a formula for Mexican adults.

\section{Participants and Methods}

The present cross-sectional study recruited 60 male students aged 21 to 23 years from the Universidad Modelo, a private University in Merida, Yucatan. The participants were selected through non-probability sampling (convenience sample) from a group of students of the Faculty of Medicine. Ethical clearance was obtained from the institutional committee and the participants signed the informed consent form as per guideline.

Anthropometric measurements were recorded by the author, following international protocol (Lohman et al. 1988). Height $(\mathrm{cm})$ was measured to the nearest tenth of a centimeter using a standard stadiometer with platform (Seca, Germany). Body weight $(\mathrm{kg})$ was recorded to the nearest $0.05 \mathrm{~kg}$ using an electronic scale (Tanita Co., Tokyo-Japan). Data of age (years), sex, and the anthropometric measurements were necessary for bioelectrical impedance analysis (BIA).

Body composition characteristics (body fat mass or FM and fat free mass or FFM) were estimated using a multifrequency whole body bioelectrical impedance (BI) analyzer Tanita MC 180 MA (Tanita Co., Tokyo-Japan), following a standard protocol (BIA 1994) and manufacturer's instruction. The equipment had eight pairs of tactile electrodes for foot pad and hand grips. Previous studies reported reliability of the obtained results using a similar model of Tanita (number 305) that mentioned best-fit algorithm of the device (Jebb et al. 2000). Participants were in light clothing, without jewelry and metal objects in the body. They were asked to abstain from foods (fasting for last 12 hours), moderate or vigorous exercise, alcohol consumption and any medicine intake in 24 hours prior to the evaluation. Assessment was done in the morning, between 7 and 8 A.M. Data collection was done in the University with prior appointments. The participants were apparently healthy and did not report any health complications at least in three months prior to the evaluation.

The FFM was also estimated using a formula for Mexican adults (Macias et al. 2007). FFM $(\mathrm{kg})=0.7374$ * $\left(\right.$ Height $^{2} /$ Resistance in ohms $)+0.1763 *$ (Body weight $)$ $-0.1773 *$ (Age) $+0.1198 *$ (Reactance in ohms) -2.4658 . The dimensions for age, height, and weight were years, $\mathrm{cm}$, and $\mathrm{kg}$ respectively. The resistance and reactance values obtained from the Tanita MC 180 MA (Tanita Co., Tokyo-Japan) have been used in the formula to estimate FFM. Body mass index (BMI) might be an interest for the readers that has been calculated as weight $(\mathrm{kg})$ divided by height in meter square. Evaluation of BMI-based nutritional status of adult men followed standard guideline (WHO 1995). Fat mass (FM) (kg and \%) was obtained from BIA and was also calculated from FFM and body 
weight. Fat mass index (FMI) was calculated as FM (kg) divided by height $\left(\mathrm{m}^{2}\right)$.

The SPSS ${ }^{\circledR}$ statistical software (version 15.00) was used for data analysis. Mean and standard deviation values of age, anthropometric (height, body weight, BMI) and body composition characteristics (FM and FFM) were calculated. Normality in the distribution of variables followed the principle of Shapiro-Wilk test ( $p>0.05)$. The values of FFM, obtained directly from the BIA and that derived from the formula (Macias et al. 2007) were compared and statistical tests to find agreement between the estimates and proportional bias were run. Intraclass correlation coefficient, t-test (one sample and paired), linear regression analysis, and Bland-Altman plots (Bland and Altman 1986) were used to estimate the agreement and bias. Level of significance was set at $\alpha=0.05$ in all analyses.

\section{Results}

The participants were young adult men (age $21.88 \pm 0.85$ years). Mean value of height $(167.63 \mathrm{~cm})$ ranged between $159.0 \mathrm{~cm}$ and $188.0 \mathrm{~cm}$. Mean value of body weight $(62.65$ $\mathrm{kg})$ also had wide ranging minimum $(48.10 \mathrm{~kg})$ and maximum $(80.70 \mathrm{~kg})$ values. The participants had moderate $\operatorname{BMI}\left(22.59 \pm 2.69 \mathrm{~kg} / \mathrm{m}^{2}\right)$ and $23.33 \%$ of them were overweight. No participant was obese. Mean values of fat free

Table 1. Descriptive statistics of age, anthropometric and body composition characteristics of young adult male university students $(n=60)$

\begin{tabular}{llll}
\hline Variables & Mean (SD) & Minimum & Maximum \\
\hline Age (years) & $21.88(0.85)$ & 21.45 & 23.87 \\
Height (cm) & $167.63(7.17)$ & 159.00 & 188.00 \\
Weight (kg) & $62.65(8.33)$ & 48.10 & 80.70 \\
BMI (kg/m²) & $22.59(2.69)$ & 19.85 & 28.81 \\
FM_BIA (kg) & $22.21(4.89)$ & 11.71 & 33.09 \\
FM-FOR (kg) & $21.29(4.59)$ & 12.47 & 32.53 \\
FMI_BIA (kg/m²) & $0.13(0.03)$ & 0.07 & 0.19 \\
FMI_FOR (kg/m²) & $0.13(0.03)$ & 0.08 & 0.19 \\
FFM_BIA (kg) & $41.44(6.08)$ & 31.12 & 54.82 \\
FFM_FOR (kg) & $41.36(5.91)$ & 31.36 & 54.71 \\
PBF_BIA (\%) & $33.37(5.83)$ & 23.37 & 45.45 \\
PBF_FOR (\%) & $33.87(5.18)$ & 24.90 & 44.80 \\
Resistance (BIA) (ohms) & $524.82(45.99)$ & 405.00 & 645.00 \\
Reactance (BIA) (ohms) & $59.79(6.68)$ & 45.60 & 71.40 \\
\hline
\end{tabular}

SD: Standard deviation; BMI: Body mass index; FM_BIA: Fat mass (BIA-based); FM_FOR: Fat mass (formula-based) (Macias et al. 2007); FMI_BIA: Fat mass index (BIA-based); FMI_FOR (FMI formula-based) (Macias et al. 2007); FFM_BIA: Fat free mass (BIA-based); FFM_FOR: Fat free mass (formula-based) (Macias et al. 2007); PBF_BIA: Percentage Body fat (BIA-based); PBF_FOR: Body fat (\%) (formula-based) (Macias et al. 2007)

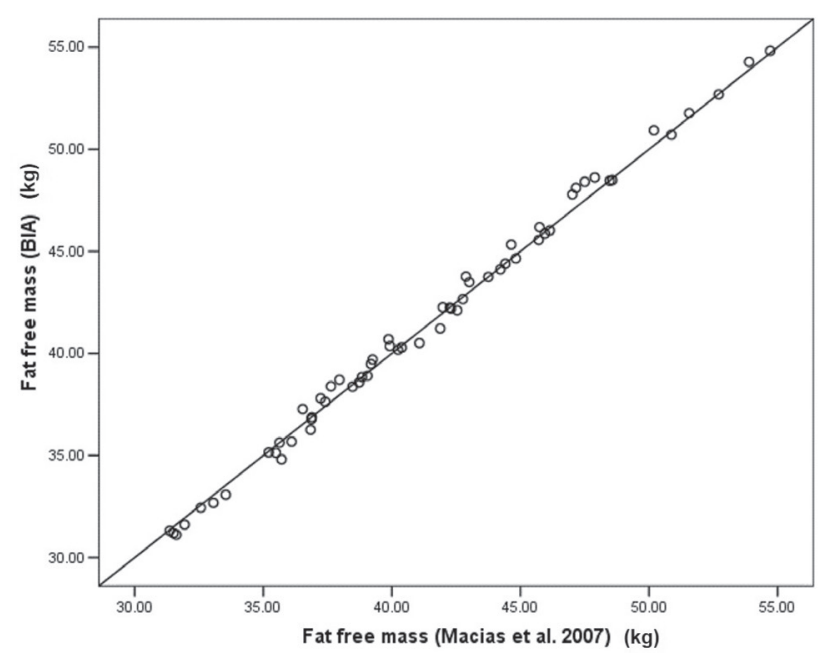

Figure 1. Scatter plot of linear regression model predicting BIA-based fat free mass (FFM) by the formula-based FFM (Macias et al. 2007).

mass (FFM) obtained directly from BIA $(41.44 \pm 6.08 \mathrm{~kg})$ was marginally different from that derived using formula (Macias et al. 2007) (41.36 $\pm 5.91 \mathrm{~kg})$. Estimated values of fat mass $(\mathrm{FM})($ BIA-based $=22.21 \pm 4.89 \mathrm{~kg}$, formulabased $=21.29 \pm 4.59 \mathrm{~kg}$ ) and fat mass index (FMI) (both BIA and formula-based $=0.13$ ) were marginally different or similar, respectively that were obtained using the two methods. Estimated mean values of percentage body fat (PBF) using BIA (33.37 $\pm 5.83 \%)$ and that derived from the FFM value obtained using formula $(33.87 \pm 5.18 \%)$ were also marginally different (Table 1 ).

Paired t-tests between BIA-based and formula-based estimates, e.g., FFM ( $\mathrm{t}=1.37, \mathrm{p}=0.18)$ and $\mathrm{PBF}(\mathrm{t}=1.43$, $\mathrm{p}=0.16)$ did not show significant differences of mean values. Intraclass correlation coefficient (ICC) between the two estimates of FFM was significant (Cronbach's alpha $=0.99, \mathrm{p}<0.0001)$. ICC of the estimated PBF using two methods also showed similar value that was calculated in case of FFM. The difference in the values of two estimates of FFM (BIA-based and formula-based) was normally distributed ( $\mathrm{p}$-value $=0.63$ ). One sample t-test for the difference of FFM values (BIA and formula-based) was not significant $(\mathrm{t}=1.37$, mean difference $-0.02, \mathrm{p}=$ 0.18). A simple linear regression model predicting BIAbased FFM from the formula-based FFM after adjusting for age and sex was significant (regression coefficient = 1.03 , standard error $=0.009, \mathrm{t}=110.79, \mathrm{p}<0.0001, \mathrm{R}^{2}$ and adjusted $\mathrm{R}^{2}=0.99$ ), showing interrelationships between the variables (Fig. 1).

In addition, BMI showed significant correlation $(\mathrm{p}<$ 0.001 ) with both BIA-based and formula-based FM ( $\mathrm{r}$ $=0.52)$, FFM $(r=0.60)$, and FMI $(r=0.60)$. However, correlation between BMI and PBF (either BIA-based or 


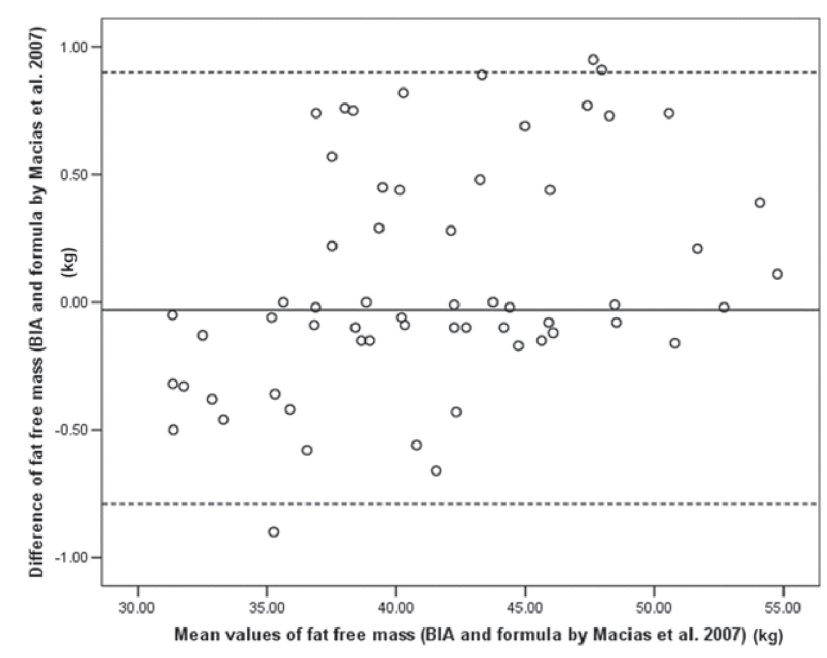

Figure 2. Bland-Altman plot of BIA-based and formula-based (Macias et al. 2007) fat free mass.

formula-based) was not significant ( $\mathrm{p}$-value $=0.40$ ).

The result of Bland-Altman test for agreement or proportional bias between the estimated FFM using two methods (BIA and formula-based) is presented in Fig. 2. In the resulting scatter plot, $\mathrm{Y}$ axis shows the difference of two paired FFM estimates (BIA and formula-based) and $\mathrm{X}$ axis presents the average of the measures. Mean value of the difference of paired estimates of FFM (BIA and formula-based) (Y axis) was -0.02 that had minimum $(-0.90)$ and maximum $(0.95)$ values, standard error of estimate 0.06 , and standard deviation 0.44 . The average of the measures (BIA and formula-based FFM) in the X axis had mean $(41.40 \mathrm{~kg})$, minimum $(31.34 \mathrm{~kg})$, maximum $(54.77 \mathrm{~kg})$ values, standard error of estimate (0.77), and standard deviation (5.99). The Bland-Altman plot shows almost all data points lie within 95\% confidence interval limits and the points were equally distributed above and below the line of mean difference (upper limit $=0.90$, lower limit $=-0.89)($ Fig. 2) that indicates no proportional bias in the estimates of FFM using two methods.

A linear regression analysis using the difference of FFM values as dependent variable and the average of the measurements as the independent variable showed no significant interrelationship $(\mathrm{t}=1.52$, $\mathrm{p}$-value 0.14 , and standard error 0.002) that accepted the null hypothesis. Age was adjusted in the model and regression coefficient was close to zero (0.003) with $95 \%$ confidence interval limits (lower -0.001, upper 0.008) that indicated no proportional bias and appeared to have an agreement between the measures (estimated FFM using BIA and that derived from formula, proposed by Macias et al. 2007).

\section{Discussion}

It is evident from the present study that the prediction formula proposed by Macias et al. (2007) to estimate FFM using the resistance and reactance values of BIA is reproducible and that has been successfully used in the present study. No significant difference between the BIA-based and formula-based FFM values was observed in the present study. A consistent agreement between the estimates was clear from correlation coefficients, regression models, and Bland-Altman plot. These results confirm the reliability of the use of resistance and reactance values to estimate body composition characteristics when the in-built algorithm of the BIA device to estimate the same is not known. The participants were young adults and values of their body composition characteristics were wide ranging: FFM (31 to $55 \mathrm{~kg}$ ) and PBF (23 to $45 \%$ ). Interestingly, BMI of the participants was not very high in the sample (mean value $22.59 \mathrm{~kg} / \mathrm{m}^{2}$ ). In addition, BMI showed significant correlation with FM, FFM, and FMI (height adjusted FM) but not with PBF. The results indicate body fat in kilogram (FM) and height adjusted values (FMI) are more important indicators of adiposity than a generalized value of PBF in relation to BMI. Considering high prevalence of BMI-based overweight and obesity in Mexican adults as reported earlier (Gutiérrez et al. 2013), the present sample of young adults $(n=60)$ showed relatively low frequency of overweight (23\%) and nobody was obese. This result may be due to a nonprobability sample that neither represent the population of this age-group from Merida, nor the State of Yucatan. In the previous study from Sonora, a northern Mexican State, mean values of BMI were also not very high (men $26.2 \mathrm{~kg} / \mathrm{m}^{2}$, women $\left.25.4 \mathrm{~kg} / \mathrm{m}^{2}\right)$ in the sample of 20 to 50-year-old adults (Macias et al. 2007).

Mexico is a country with huge ethnic and genetic diversity (Moreno-Estrada et al. 2014). We understand that human biology of the populations in the north of the country is different from that in the Southern Peninsular Mexico. Mean values of BIA-based PBF (25.8\%) and FFM $(57.9 \mathrm{~kg})$ among adult male participants in the previous report (Macias et al. 2007) were different from that recorded in the present study (PBF 33.37\%, FFM $41.44 \mathrm{~kg}$ ). The adult men in the present study showed higher PBF and lower FFM in comparison with that reported in the previous study (Macias et al. 2007). This may be interpreted as differences in body composition characteristics between the two sub-populations of Mexico.

A standard multifrequency whole body bioelectrical impedance (BI) analyzer Tanita MC 180 MA (Tanita Co. Tokyo-Japan) was used in the present study that was reported to be similar in the reliability of another model 
(Tanita 305) used in a previous study (Jebb et al. 2000). Body composition characteristics were evaluated using a different equipment (Model BIA-103, RJL Systems Detroit, MI) in the previous study by Macias et al. (2007). When the in-built software programs for body composition assessment are not known, it seems that the resistance and reactance values obtained from the equipment used in the present study worked satisfactorily. The resistance and reactance-based prediction formula to estimate FFM for adults (Macias et al. 2007) is pioneering of its kind in Mexico. In their study, the authors cross-validated the results obtained using the equation with reference to results from the air displacement plethysmography (ADP). Mean values of BIA-based and ADP-based FFM were same $(57.9 \mathrm{~kg})$ among men in that study. In the present study, mean value of BIA-based FFM (41.44 kg) was marginally different from the derived one obtained using the resistance and reactance values applied in the prediction equation $(41.36 \mathrm{~kg}$ ) proposed earlier (Macias et al. 2007). However, the difference in the mean values was not significant $(\mathrm{p}>0.05)$ as evaluated using paired $\mathrm{t}$-test.

Dual-energy X-ray absorptiometry (DEXA or DXA) is considered as a gold standard for the evaluation of body composition characteristics (Laskey 1996; Scafoglieri and Clarys 2018). Magnetic resonance imaging and DXA are highly precise methods to estimate body composition characteristics (Taing et al. 2017). However, the instruments are expensive, invasive, not portable, and time consuming for the evaluation process. In large-scale surveys, BIA provides satisfactory results and advanced models are capable of segmental analysis of body composition: whole body and regional (extremities). Studies reported higher reliability of the results from whole body BIA than segmental BIA when the values were compared with the results obtained using DXA (Bracco et al. 1996; Demura et al. 2004; Erselcan et al. 2000; Ricciardi and Talbot 2007). In this background, BIA-based prediction formula for FFM on a two-compartment model was reported to be accurate, precise, and free of bias (Macias et al. 2007).

A study from Mexico among 86 postmenopausal women aged 42 to 74 years with metabolic syndrome (Balas-Nakash et al. 2010), estimated body fat using DXA and compared the results with the derived values using the formulas proposed in a study based on the data of National Health and Nutrition Examination Survey III (NHANES III) (Sun et al. 2005) of the U.S.A and by another study from Mexico (Macias et al. 2007). The DXA-based PBF (44.54\%) had different levels of agreement (Bland-Altman plot) with the values derived from the formula proposed in a NHANES III-based study (41.93\%) (Sun et al. 2005) and that estimated using the equation proposed by Macias et al. 2007 (43.32\%). The NHANES III-based formula and prediction equation by Macias et al. 2007 underestimated
PBF. However, the report (Balas-Nakash et al. 2010) raised doubt about the use of prediction equation to estimate FFM that was proposed by Macias et al. 2007 because age of many women participants in that study was higher than 50 years. In the reference study (Macias et al. 2007), age of women participants was between 22 and 48 years.

A study from China, developed BIA-based prediction equations to estimate FFM for adult men and women using two different BI instruments (hand-to-hand and hand-to-foot) and DXA was the criterion method (Lu et al. 2016). Both types of BIA-based prediction equations demonstrated similar reliability and the hand-to-hand BIA model was found to be more practical for the assessment of body composition characteristics. The study suggested importance of separate prediction equations for men and women. Another study among indigenous Australian adults aged 36 to 60 years developed prediction equation to estimate FFM and the reference method was DXA (Hughes et al. 2015).

A study from Brazil reported that mean values of BIA-based PBF (38.0\%) and formula-based PBF (38.7\%) were similar; BIA overestimated PBF in overweight and underestimated the same among obese adult women (Pimentel et al. 2010). The formula was developed in an earlier study (Segal et al. 1988). Low sensitivity of BIA to identify individuals with high BMI and body fat was reported by several authors (Macias et al. 2007; Pimentel et al.2010). Another study among adults of 18 to 64 years of age from Chile (Schifferli Castro and Zuniga 2017) compared BIA-based estimate of FFM with that derived from a prediction formula for FFM reported earlier (Schifferli et al. 2011). The previous study (Schifferli et al. 2011) developed prediction equation for BIA-based FFM, taking DEXA as the criterion or reference method. BIA overestimated PBF in men and underestimated in women compared to the corresponding values obtained using the prediction formula (Schifferli Castro and Zuniga 2017). Therefore, it is evident that BIA-based estimates of FFM may be different in men and women and vary with the levels of BMI and body fatness. This raised concern for separate formulas for men and women and selection of a precise criterion method like DXA. In addition, this issue reflects the limitation of the use of the BIA-based estimates where the in-built algorithm of the device (that varies with the models also) is not known.

A cross-sectional study among university students aged 18 to 24 years from Colombia developed single-frequency BIA-based prediction equations for total body water and FFM, where BI spectroscopy for three compartment model was used as the criterion method (Caicedo-Eraso et al. 2019). In both methods, resistance and reactance values were used. Estimated values of total body water and FFM that were obtained using the two methods did not show 
significant differences. The results of regression models showing low standard error of estimates and increase in $\mathrm{R}^{2}$ were like that reported in Macias et al. 2007.

A study from the U.S. (Wada and Tekin 2007) used BIA-based FFM data from NHANES III and applied the prediction equations reported earlier from the same country for men and women to derive FFM (Sun et al. 2003). The study reported huge number of prediction formulas for FFM and lean body mass (LBM). The authors (Wada and Tekin 2007) also cited articles to show the conversion of LBM to FFM using formulas. Therefore, prediction formulas for FFM had received better technical precision.

The prediction formula by Macias et al. 2007 is common for adult men and women. Another study also reported single prediction equation to estimate FFM for adult men and women aged 20 to 94 years of age (Kyle et al. 2001). Separate prediction formulas for men and women were reported from the U.S. (Sun et al. 2003); validation and cross-validation samples of whites and blacks were taken into consideration. A report from China also reported separate equations for adult men and women ( $\mathrm{Lu}$ et al.2016). Higher adiposity in women compared to men was reported in previous studies from Nigeria (Mbada et al. 2015) and Mexico (Macias et al. 2007). High correlation between BIA-based PBF and skinfold-based values was observed instead of overestimation of PBF by the former method (Mbada et al. 2015).

Accuracy of the results obtained using BIA depends on the specific algorithm of the device (Jaffrin and Bousbiat 2014). Therefore, validation and cross-validation of the results obtained from an equipment are important before use. Comparison of the results with that obtained through more precise method like DXA is important. In spite of several limitations of the use of BIA as mentioned before (Coppini et al. 2005), the advantages include portability, safety, non-invasive nature of application, less intraobserver error in the estimation of body composition characteristics, segmental analysis of body fat and fat free mass, relatively moderate cost, and reliable results etc. However, care should be taken for the hydration factor and body geometry of severely obese individuals, variation of body water during menstruation and other health conditions. Another important issue will be the use of the terms FFM and LBM that are not interchangeable. The LBM consists of FFM and essential body fat that may vary between 2\% and 10\% (Scafoglieri and Clarys 2018; Segal et al. 1988).

In this background, it is justified to mention the limitations of the present study and new call for improved research. In the present study, the term FFM has been used to maintain uniformity with the reference study (Macias et al. 2007). Previous reports on the use of BIA, authors mentioned LBM prediction instead of FFM (Tagliabue et al. 2001). Another report also cited articles on how to convert prediction formulas for LBM to FFM (Wada and Tekin 2007). This issue should be considered in future research. Other limitations of the present study were nonprobability sampling, relatively small sample size from a short age-range of 21 to 23-year-old adult men. Neither of the two samples (Macias et al. 2007 and the present study) were probabilistic. The present report shows the results of male participants. Therefore, sample size and age might be the other important factors that call for future research to explore the results in wider age-group and in the representative samples of the populations from different regions of Mexico where individuals with BMI-based nutritional status of normal, overweight, and obesity will be present. It is also important to estimate the differential agreement and bias of the obtained values of FFM using two methods at the levels of BMI. Perhaps in future research, separate formulas for men and women will give more precise results as reported in other studies that were also compared with the results obtained through DXA (Sun et al. 2003). Separate prediction equations for men and women may show differential degree of agreement between the BIA-based FFM and the corresponding value derived from the resistance and reactance-based equations.

Despite such limitations, the present study showed significant concordance of the estimated values of FFM using BIA with that estimated one using the prediction formula (resistance and reactance value-based). The results further established the reproducibility of the prediction formula for Mexican adults proposed earlier (Macias et al. 2007).

From the above discussion, it seems that the estimation of FFM using the BIA has certain limitations because the in-built algorithm of the device is not known and that also may vary in different models. Moreover, the formulas used in the program of the device may differ by age, sex, activity patterns, and population backgrounds. On the other hand, the prediction formulas to estimate FFM using the resistance and reactance values obtained from BIA has several advantages over the problems of unknown algorithm of the device. Studies reported agreement of the results obtained using such formulas with those obtained using a gold standard method like DXA. However, some reports are also available on the disagreement of results (over and underestimation of FFM) at the levels of high BMI (overweight and obesity) and body fat. Differential agreements were also found in men and women.

In conclusion, the present study has shown significant agreement of the estimated FFM using the bioelectrical impedance analyzer (Tanita MC 180 MA, Tanita Corporation, Tokyo, Japan) with the estimated FFM using the resistance and reactance values obtained from the BIA that has been used in a prediction formula proposed 
earlier for Mexican adults (Macias et al. 2007).

\section{Acknowledgements}

The author thankfully acknowledges help and cooperation of the colleagues and participants from the Faculty of Medicine of the Universidad Modelo in Merida, Yucatan. The author is also thankful to a colleague of the Autonomous University of Yucatan (UADY) for lending the bioelectrical impedance analyzer during field work. The author declares no conflict of interest.

\section{References}

Balas-Nakash M, Legorreta-Legorreta J, Rodríguez-Cano A, Aguilera-Pérez R, Perichart-Perera O (2010) Validación del uso de ecuaciones para estimar la composición corporal por análisis de impedancia bioeléctrica en mujeres postmenopáusicas con síndrome metabólico. Rev Invest Clín 62(6):538-545

BIA (No author listed) (1994) Bioelectrical impedance analysis in body composition measurement. Proceedings of a National Institutes of Health Technology Assessment Conference. Bethesda, Maryland, December 12-14, 1994. Am J Clin Nutr 64:387S-532S.

Bland JM, Altman DG (1986) Statistical methods for assessing agreement between two methods of clinical measurement. Lancet 1:307-310.

Bohm A, Heitmann BL (2013) The use of bioelectrical impedance analysis for body composition in epidemiological studies. Eur J Clin Nutr 67:S79-S85.

Bracco D, Thiebaud D, Chiolero RL, Landry M, Burckhardt P, Schutz Y (1996) Segmental body composition assessed by bioelectrical impedance analysis and DEXA in humans. J Appl Physiol 81(6):2580-2587.

Caicedo-Eraso JC, Gonzalez-Correa CH, Gonzalez-Correa CA, Gallagher D (2019) Bioelectrical impedance analysis, hydrometry and hydrodensitometry for body composition assessment in adult Colombian women. J Phys Conf Ser (3rd Latin-American Conference on Bioimpedance) 1272:012002.

Coppini LZ, Waitzberg DL, Campos ACL (2005) Limitations and validation of bioelectrical impedance analysis in morbidly obese patients. Curr Opin Clin Nutr 8(3):329-332.

Demura S, Sato S, Kitabayashi T (2004) Percentage of total body fat as estimated by three automatic bioelectrical impedance analyzers. J Physiol Anthropol Appl Human Sci 23(3):93-99.

Dwyer JT, Gallo JJ, Reichel W (1993) Assessing nutritional status in elderly patients. Am Fam Physician 47(3):613-
620.

Erselcan T, Candan F, Saruhan S, Ayca T (2000) Comparison of body composition analysis methods in clinical routine. Ann Nutr Metab 44(5-6):243-248.

Gutiérrez JP, Rivera-Dommarc, J, Shamah-Levy T, Villalpando-Hernández S, Franco A, Cuevas-Nasu L, Romero-Martínez M, Hernández-Ávila M (2013) Encuesta Nacional de Salud y Nutrición 2012. Resultados nacionales (2a ed.) Cuernavaca, México: Instituto Nacional de Salud Pública.

Hughes, JT, Maple-Brown LJ, Piers LS, Meerkin J, O’Dea K, Ward LC (2015) Development of a single-frequency bioimpedance prediction equation for fat-free mass in an adult Indigenous Australian population. Eur J Clin Nutr 69(1):28-33.

Jaffrin MY, Bousbiat S (2014) Accuracy of plantar electrodes compared with hand and foot electrodes in fat-free-mass measurement. J Healthc Eng 5(2):123-144.

Jebb SA, Cole TJ, Doman D, Murgatroyd PR, Prentice AM (2000) Evaluation of the novel Tanita body-fat analyser to measure body composition by comparison with a four-compartment model. Br J Nutr 83(2):115-22.

Khalil SF, Mohktar MS, Ibrahim F (2014) The theory and fundamentals of bioimpedance analysis in clinical status monitoring and diagnosis of diseases. Sensors 14:1089510928.

Kyle UG, Genton L, Karsegard L, Slosman DO, Pichard C (2001) Single prediction equation for bioelectrical impedance analysis in adults aged 20-94 yrs. Nutrition 17:248-283.

Laskey MA (1996) Dual-energy X-ray absorptiometry and body composition. Nutrition 12(1):45-51.

Lohman TG, Roche AF, Martorell R (1988) Anthropometric Standardization Reference Manual. Champagne, IL.

Lu HK, Chiang LM, Chen YY, Chuang CL, Chen KT, Dwyer GB, Hsu YL, Chen CH, Hsieh KC (2016) Hand-to-hand model for bioelectrical impedance analysis to estimate fat free mass in a healthy population. Nutrients 8(10):654.

Macias N, Alemán-Mateo H, Esparza-Romero J, Valencia ME (2007) Body fat measurement by bioelectrical impedance and air displacement plethysmography: a cross-validation study to design bioelectrical impedance equations in Mexican adults. Nutr J 6:18.

Mbada CE, Akintayo ND, Johnson OE, Dada OO (2015) Correlates, predictors, reference ranges and agreement between percent body fat measured using bioelectric impedance analysis and skinfold-thickness measurements in young Nigerian adults. Arch Physiother Glob Res 19(4):7-20.

Moreno-Estrada A, Gignoux CR, Fernández-López JC, Zakharia F, Sikora M, Contreras AV, Acuña-Alonzo V, Sandoval K, Eng C, Romero-Hidalgo S, Ortiz Tello P, Robles V, et al. (2014) The Genetics of Mexico recapitu- 
lates Native American substructure and affects biomedical traits. Science 344(6189):1280-1285.

Pimentel GD, Bernhard AB, Frezza MP, Rinaldi AM, Burini RC (2010) Bioelectric impedance overestimates the body fat in overweight and underestimates in Brazilian obese women: a comparation with Segal equation 1. Nutr Hosp 25(5):741-745.

Ramírez E, Valencia ME, Bourges H, Espinosa T, MoyaCamarena SY, Salazar G, Aléman-Mateo H (2012) Body composition prediction equations based on deuterium oxide dilution method in Mexican children: a national study. Eur J Clin Nutr 66(10):1099-1103.

Ricciardi R, Talbot LA (2007) Use of bioelectrical impedance analysis in the evaluation, treatment, and prevention of overweight and obesity. J Am Acad Nurse Pract 19(5):235-241.

Scafoglieri A, Clarys JP (2018) Dual energy X-ray absorptiometry: gold standard for muscle mass? Letter to the Editor. J Cachexia Sarcopeni Muscle 9:786-787.

Schifferli I, Carrasco F, Inostroza J (2011) Formulación de una ecuación para predecir la masa grasa corporal a partir de bioimpedanciometría en adultos en un amplio rango de edad e índice de masa corporal. Rev Med Chile 139:1534-1543.

Schifferli Castro I, Zuniga ML (2017) Behavior of a new equation using bio-impedancemetry to predict body fat in Chilean adults. Obes Control Ther 4(2):1-7.

Sebo P, Herrmann FR, Haller DM (2017) Accuracy of anthropometric measurements by general practitioners in overweight and obese patients. BMC Obes 4:23.

Segal KR, Van Loan M, Fitzgerald PI, Hodgdon JA, Van Itallie TB (1988) Lean body mass estimation by bioelectrical impedance analysis: a four-site cross-validation study. Am J Clin Nutr 47(1):7-14.

Sun SS, Chumlea WC, Heymsfield SB, Lukaski HC, Schoeller
D, Friedl K, Kuczmarski RJ, Flegal KM, Johnson CL, Hubbard VS (2003) Development of bioelectrical impedance prediction equations from body composition with the use of a multicomponent model for use in epidemiologic surveys. Am J Clin Nutr 77:331-340.

Sun G, French CR, Martin GR, Younghusband B, Green RC, Xie YG, Mathews M, Barron JR, Fitzpatrick DG, Gulliver $\mathrm{W}$, Zhang H (2005) Comparison of multifrequency bioelectrical impedance analysis with dual-energy X-ray absorptiometry for assessment of percentage body fat in a large, healthy population. Am J Clin Nutr 81:74-78.

Tagliabue A, Andreoli A, Comelli M, Bertoli S, Testolin G, Oriani G, De Lorenzo A (2001) Prediction of lean body mass from multifrequency segmental impedance: influence of adiposity. Acta Diabetol 38:93-97

Taing KY, Farkuoh ME, Moineddin R, Tu JV, Jha P (2017) Comparative associations between anthropometric and bioelectric impedance analysis derived adiposity measures with blood pressure and hypertension in India: a cross-sectional analysis. BMC Obes 4:37.

Wada R, Tekin E (2007) Body Composition and Wages. NBER Working Paper No. 13595. National Bureau of Economic Research. Cambridge, MA.

WHO (1995) Physical status: the use and interpretation of anthropometry. Report of a WHO expert committee. Technical report series no. 854. Geneva: World Health Organization. From: <http://whqlibdoc.who. int/trs/WHO_TRS_854.pdf $\geq$ (Retrieved on $5^{\text {th }}$ October 2020).

Yamashita K, Kondo T, Osugi S, Shimokata K, Maeda K, Okumura N, Matsudaira K, Shintani S, Muramatsu T, Matsushita K, Murohara T (2012) The significance of measuring body fat percentage determined by bioelectrical impedance analysis for detecting subjects with cardiovascular disease risk factors. Circ J 76(10):2435-2442. 